TRANSACTIONS OF THE

AMERICAN MATHEMATICAL SOCIETY

Volume 365, Number 3, March 2013, Pages 1533-1550

S 0002-9947(2012)05674-1

Article electronically published on September 27, 2012

\title{
$h$-VECTORS OF SIMPLICIAL CELL BALLS
}

\author{
SATOSHI MURAI
}

\begin{abstract}
A simplicial cell ball is a simplicial poset whose geometric realization is homeomorphic to a ball. Recently, Samuel Kolins gave a series of necessary conditions and sufficient conditions on $h$-vectors of simplicial cell balls, and characterized them up to dimension 6. In this paper, we extend Kolins' results. We characterize all possible $h$-vectors of simplicial cell balls in arbitrary dimension.
\end{abstract}

\section{INTRODUCTION}

A simplicial poset is a finite poset $P$ with a minimal element $\hat{0}$ such that every interval $[\hat{0}, \sigma]$ for $\sigma \in P$ is a Boolean algebra. A typical example of a simplicial poset is the face poset of a simplicial complex, but not all simplicial posets come from simplicial complexes. Simplicial posets are CW-posets. Thus for any simplicial poset $P$ there is a regular $\mathrm{CW}$-complex $\Gamma(P)$ whose face poset is isomorphic to $P$ (see [Bj1, pp. 8-9]). A simplicial poset $P$ (or a CW-complex $\Gamma(P)$ ) is called a simplicial cell d-ball (respectively d-sphere) if $\Gamma(P)$ is homeomorphic to a $d$-ball (respectively $d$-sphere). Kolins $[\mathrm{Ko}$ studied $h$-vectors of simplicial cell balls and gave a characterization of $h$-vectors of simplicial cell balls up to dimension 6 . In this paper, we extend the results of Kolins and give a complete characterization of $h$-vectors of simplicial cell balls.

Let $P$ be a simplicial poset. We say that an element $\sigma \in P$ has $\operatorname{rank} i$, denoted $\operatorname{rank} \sigma=i$, if $[\hat{0}, \sigma]$ is a Boolean algebra of rank $i$. The dimension of $P$ is

$$
\operatorname{dim} P=\max \{\operatorname{rank} \sigma: \sigma \in P\}-1 .
$$

Let $d=\operatorname{dim} P+1$ and let $f_{i}=f_{i}(P)$ be the number of elements $\sigma \in P$ having rank $i+1$ for $i=-1,0, \ldots, d-1$. Thus $f_{i}$ is the number of $i$-dimensional cells of $\Gamma(P)$. The vector $f(P)=\left(f_{-1}, f_{0}, \ldots, f_{d-1}\right)$ is called the $f$-vector (face vector) of $P$. We define the $h$-vector $h(P)=\left(h_{0}(P), h_{1}(P), \ldots, h_{d}(P)\right) \in \mathbb{Z}^{d+1}$ of $P$ by the relation

$$
\sum_{i=0}^{d} f_{i-1}(t-1)^{d-i}=\sum_{i=0}^{d} h_{i}(P) t^{d-i} .
$$

Then knowing $f(P)$ is equivalent to knowing $h(P)$.

On face vectors of simplicial cell spheres, Stanley [St1] and Masuda [Ma] proved the following result, which characterizes all possible $h$-vectors of simplicial cell spheres.

Received by the editors May 31, 2011 and, in revised form, July 19, 2011.

2010 Mathematics Subject Classification. Primary 05E45, 52B05; Secondary 13F55.

(C) 2012 American Mathematical Society Reverts to public domain 28 years from publication 
Theorem 1.1 (Stanley, Masuda). Let $\mathbf{h}=\left(h_{0}, h_{1}, \ldots, h_{d}\right) \in \mathbb{Z}^{d+1}$. Then $\mathbf{h}$ is the $h$-vector of a simplicial cell $(d-1)$-sphere if and only if it satisfies the following conditions:

(1) $h_{0}=h_{d}=1$ and $h_{i}=h_{d-i}$ for $i=1,2, \ldots, d-1$.

(2) $h_{i} \geq 0$ for $i=0,1, \ldots, d$.

(3) If $h_{n}=0$ for some $1 \leq n \leq d-1$, then $\sum_{k=0}^{d} h_{k}$ is even.

For a vector $\mathbf{h}=\left(h_{0}, h_{1}, \ldots, h_{d}\right) \in \mathbb{Z}^{d+1}$, we define $\partial \mathbf{h}=\left(\partial h_{0}, \partial h_{1}, \ldots, \partial h_{d-1}\right) \in$ $\mathbb{Z}^{d}$ by

$$
\partial h_{i}=\left(h_{0}+h_{1}+\cdots+h_{i}\right)-\left(h_{d}+h_{d-1}+\cdots+h_{d-i}\right)
$$

for $i=0,1, \ldots, d-1$. It is known that if $\mathbf{h}$ is the $h$-vector of a simplicial cell $(d-1)$-ball $P$, then $\partial \mathbf{h}$ is the $h$-vector of the boundary sphere of $P$. In this paper, we prove the next result, which characterizes all possible $h$-vectors of simplicial cell balls.

Theorem 1.2. Let $\mathbf{h}=\left(h_{0}, h_{1}, \ldots, h_{d}\right) \in \mathbb{Z}^{d+1}$. Then $\mathbf{h}$ is the h-vector of $a$ simplicial cell $(d-1)$-ball if and only if it satisfies the following conditions:

(1) $h_{0}=1, h_{d}=0$ and $h_{k} \geq 0$ for $k=1,2, \ldots, d-1$.

(2) $\partial h_{k} \geq 0$ for $k=0,1, \ldots, d-1$.

(3) If $d$ is odd and $\partial h_{n}=0$ for some $1 \leq n \leq d-2$, then $\sum_{k=0}^{d} h_{k}$ is even.

(4) If $\partial h_{n}=0$ for some $1 \leq n \leq d-2$, then

$$
h_{k}+h_{k-1}+\cdots+h_{k-n+1} \geq \partial h_{k} \quad \text { for } k=n, n+1, \ldots, d-1 .
$$

(5) If $\partial h_{i}=0$ and $h_{j}=0$ for some positive integers $i$ and $j$ with $i+j \leq d$, then $\sum_{k=0}^{d} h_{k}$ is even.

(6) Suppose $\partial h_{n}=0$ for some $1 \leq n<\frac{d}{2}$. If $\left(h_{\ell}+h_{\ell-1}+\cdots+h_{\ell-n+1}\right)-\partial h_{\ell}<n$ for some $n \leq \ell \leq d-n$, then $\sum_{k=0}^{d} h_{k}$ is even.

(7) Suppose $\partial h_{i}=0$ and $h_{j}=0$ for some integers $i$ and $j$ with $0<i<\frac{d}{2}$ and $d-i<j<d$. If $\partial h_{\ell} \leq \ell$ for some $\ell \leq d-j$, then $\sum_{k=0}^{d} h_{k}$ is even.

Note that conditions (5), (6) and (7) are unnecessary when $d$ is odd. We also note that some special cases of the above theorem are due to Kolins [Ko. For necessity, (1), (2), (3) and some special cases of (4), (5) and (7) (the condition (4) for $n=1$, the condition (5) for $i=1,2$ and $j=1$, and the condition (7) for $\ell=1$ ) were proved in [ Ko, Sections 3,4 and 5]. Also, when $\partial \mathbf{h}$ is positive or $\sum_{k=0}^{d} h_{k}$ is even, the sufficiency of the theorem was proved in [Ko, Theorem 16]. In particular, for even-dimensional simplicial cell balls, the sufficiency of the theorem is due to Kolins.

A simplicial poset $P$ (or a $\mathrm{CW}$-complex $\Gamma(P)$ ) is called a simplicial cell decomposition of a topological space $X$ if $\Gamma(P)$ is homeomorphic to $X$. It was proved in $[\mathrm{Mu}$. that face vectors of simplicial cell decompositions of some manifolds without boundary, such as products of spheres and real projective spaces, are characterized by conditions similar to those in Theorem 1.1. It would be of interest to find manifolds $M$ with boundary such that face vectors of simplicial cell decompositions of $M$ can be characterized by conditions similar to those in Theorem 1.2 .

This paper is organized as follows. In Section 2, we prove the necessity of Theorem 1.2 by using the face ring of a simplicial poset. In Section 3, we prove the sufficiency of Theorem 1.2 by using constructibility and shellability. 


\section{Proof OF NECESSITY}

In this section, we prove the necessity of Theorem 1.2. To prove this, we need the face ring of a simplicial poset introduced by Stanley [St1]. We assume familiarity with commutative algebra theory.

We recall some basic notation on commutative algebra. Let $K$ be an infinite field, $R=R_{0} \oplus R_{1} \oplus \cdots$ a finitely generated commutative graded $K$-algebra with $R_{0}=K$, where $R_{k}$ is the graded component of $R$ of degree $k$, and let $M$ be a finitely generated graded $R$-module. The Hilbert series $H_{M}(t)$ of $M$ is the formal power series $H_{M}(t)=\sum_{k=0}^{\infty}\left(\operatorname{dim}_{K} M_{k}\right) t^{k}$. The Krull dimension of $M$, denoted $\operatorname{dim} M$, is the minimal $m$ for which there exist homogeneous elements $\theta_{1}, \ldots, \theta_{m} \in R$ of positive degrees such that $M /\left(\theta_{1}, \ldots, \theta_{m}\right) M$ is a finite-dimensional $K$-vector space. When $\operatorname{dim} M=d$, a sequence of homogeneous elements $\theta_{1}, \ldots, \theta_{d} \in R$ of positive degrees such that $M /\left(\theta_{1}, \ldots, \theta_{d}\right) M$ is a finite-dimensional $K$-vector space is called a homogeneous system of parameters (h.s.o.p. for short) of $M$. Moreover, if $\theta_{1}, \ldots, \theta_{d}$ have degree 1 , then we call $\theta_{1}, \ldots, \theta_{d}$ a linear system of parameters (l.s.o.p. for short) of $M$. We say that $M$ is Cohen-Macaulay if, for every (equivalently some) h.s.o.p. $\theta_{1}, \ldots, \theta_{d}$ of $M$, one has that $\theta_{i}$ is a non-zero divisor of $M /\left(\theta_{1}, \ldots, \theta_{i-1}\right) M$ for all $i=1,2, \ldots, d$. Also, $R$ is said to be Cohen-Macaulay if it is a CohenMacaulay $R$-module.

Let $P$ be a $(d-1)$-dimensional simplicial poset and let $A=K\left[x_{\sigma}: \sigma \in P \backslash\{\hat{0}\}\right]$ be the polynomial ring over an infinite field $K$ in indeterminates indexed by the elements in $P \backslash\{\hat{0}\}$. We define the grading of $A$ by $\operatorname{deg} x_{\sigma}=\operatorname{rank} \sigma$. The face ring of $P$ is the quotient ring $K[P]=A / I_{P}$, where $I_{P}$ is the ideal generated by the following elements:

- $x_{\sigma} x_{\tau}$ if $\sigma, \tau \in P$ have no common upper bounds in $P$.

- $x_{\sigma} x_{\tau}-x_{\sigma \wedge \tau} \sum_{\rho} x_{\rho}$, where the summation runs over all the minimal upper bounds of $\sigma$ and $\tau$ and where $\sigma \wedge \tau$ is the meet (largest lower bound) of $\sigma$ and $\tau$, otherwise. (We consider $x_{\sigma \wedge \tau}=1$ if $\sigma \wedge \tau=\hat{0}$.)

Note that, for all $\sigma, \tau \in P$ such that $\sigma$ and $\tau$ have a common upper bound $\rho$, there is the unique largest lower bound of $\sigma$ and $\tau$ since $[\hat{0}, \rho]$ is a Boolean algebra. Also, since generators of $I_{P}$ are homogeneous, the ring $K[P]$ is graded. In the special case when $P$ is the face poset of a simplicial complex, the ring $K[P]$ is isomorphic to the face ring (Stanley-Reisner ring) of a simplicial complex [St2, p. 53]. See [MMP, p. 467].

A simplicial poset $P$ is said to be Cohen-Macaulay if the ring $K[P]$ is CohenMacaulay. Note that this is equivalent to saying that the order complex of $P \backslash\{\hat{0}\}$ is a Cohen-Macaulay simplicial complex. On face rings of simplicial posets, the following properties are known.

- The Krull dimension of $K[P]$ is $\operatorname{dim} P+1$.

- $K[P]$ has an l.s.o.p.

- $H_{K[P]}(t)=\left(h_{0}+h_{1} t+\cdots+h_{d} t^{d}\right) /(1-t)^{d}$, where $d=\operatorname{dim} P+1$ and where $\left(h_{0}, h_{1}, \ldots, h_{d}\right)=h(P)$.

- If $\Gamma(P)$ is homeomorphic to a ball or a sphere, then $P$ is Cohen-Macaulay.

See [St1, pp. 325-326] for the first three properties. The last property follows from a topological criterion of Cohen-Macaulay simplicial complexes St2, II, Corollary 4.2 and Proposition 4.3]. 
A subset $I$ of a simplicial poset $P$ is said to be an order ideal of $P$ if $\sigma \in I$ and $\tau \leq \sigma$ imply $\tau \in I$. Thus an order ideal of a simplicial poset is again a simplicial poset. For a simplicial poset $P$ and elements $\sigma_{1}, \ldots, \sigma_{k} \in P$, we write $\left\langle\sigma_{1}, \ldots, \sigma_{k}\right\rangle$ for the order ideal of $P$ generated by $\sigma_{1}, \ldots, \sigma_{k}$; in other words,

$$
\left\langle\sigma_{1}, \ldots, \sigma_{k}\right\rangle=\left\{\tau \in P \text { : there is } 1 \leq j \leq k \text { such that } \tau \leq \sigma_{j}\right\} .
$$

For elements $f_{1}, \ldots, f_{m}$ in a ring $A$, we write $\left(f_{1}, \ldots, f_{m}\right)$ for the ideal of $A$ generated by $f_{1}, \ldots, f_{m}$. We often use the following obvious fact.

Lemma 2.1. Let $P$ be a simplicial poset and $A=K\left[x_{\sigma}: \sigma \in P \backslash\{\hat{0}\}\right]$. If $Q$ is an order ideal of $P$, then $A /\left(I_{P}+\left(x_{\sigma}: \sigma \notin Q\right)\right)$ is isomorphic to $K[Q]$ as a ring.

Let $P$ be a simplicial cell $(d-1)$-ball. Then each rank $d-1$ element of $P$ is covered by at most two elements. The boundary $\partial P$ of $P$ is the order ideal

$$
\partial P=\langle\sigma \in P: \operatorname{rank} \sigma=d-1, \sigma \text { is covered by exactly one element in } P\rangle .
$$

Since $\partial P$ is the face poset of the boundary cell complex of $\Gamma(P), \partial P$ is a simplicial cell $(d-2)$-sphere. Moreover, it is known that if $\mathbf{h}=\left(h_{0}, h_{1}, \ldots, h_{d}\right)$ is the $h$-vector of $P$, then $\partial \mathbf{h}=\left(\partial h_{0}, \partial h_{1}, \ldots, \partial h_{d-1}\right)$ is the $h$-vector of $\partial P$. See [Ko, Section 3]. On $h$-vectors of simplicial cell balls, the following result was proved in $[\mathrm{Ko}$, Theorem $5]$.

Theorem 2.2 (Kolins). Let $P$ be a simplicial cell $(d-1)$-ball and $\mathbf{h}=h(P)=$ $\left(h_{0}, h_{1}, \ldots, h_{d}\right)$. Then

(1) $h_{0}=1, h_{d}=0$ and $h_{k} \geq 0$ for $k=1,2, \ldots, d-1$.

(2) $\partial h_{k} \geq 0$ for $k=0,1, \ldots, d-1$.

(3) If $d$ is odd and $\partial h_{n}=0$ for some $1 \leq n \leq d-2$, then $\sum_{k=0}^{d} h_{k}$ is even.

We sketch a proof of the above theorem. The vectors $\mathbf{h}$ and $\partial \mathbf{h}$ are non-negative since $h$-vectors of Cohen-Macaulay simplicial posets are non-negative St1, Theorem 3.10]. Also, since $h_{d}(P)$ is equal to the reduced Euler characteristic of $P$ times $(-1)^{d-1}, h_{d}(P)=0$ if $P$ is a simplicial cell ball. Finally, (3) follows from Theorem 1.1 as follows: If $d$ is odd, then

$$
\partial h_{\frac{d-1}{2}}=\left(h_{0}+\cdots+h_{\frac{d-1}{2}}\right)-\left(h_{\frac{d+1}{2}}+\cdots+h_{d}\right)
$$

is equal to $\sum_{k=0}^{d} h_{k} \bmod 2$. Since $\sum_{k=0}^{d-1} \partial h_{k}$ is even by Theorem 1.1(3), by the symmetry $\partial h_{i}=\partial h_{d-1-i}$ of $\partial \mathbf{h}, \partial h_{\frac{d-1}{2}}$ must be even.

In the rest of this section, we prove that conditions (4), (5), (6) and (7) in Theorem 1.2 are necessary conditions of $h$-vectors of simplicial cell balls.

\subsection{Proof of (4).}

Lemma 2.3. Let $P$ be a $(d-1)$-dimensional simplicial poset and $h(P)=\left(h_{0}, \ldots\right.$, $\left.h_{d}\right)$. Let $K[P]=A / I_{P}$ be the face ring of $P$ and $\theta_{1}, \ldots, \theta_{d} \in A_{1}$ an l.s.o.p. of $K[P]$. If $K[P]$ is Cohen-Macaulay, then, for all integers $n>0$ and $n \leq k \leq d$, one has

$$
\operatorname{dim}_{K}\left(A /\left(I_{P}+\left(\theta_{1}, \ldots, \theta_{d-1}, \theta_{d}^{n}\right)\right)\right)_{k}=h_{k}+h_{k-1}+\cdots+h_{k-n+1} .
$$

Proof. Let $R[i]=A /\left(I_{P}+\left(\theta_{1}, \ldots, \theta_{i}\right)\right)$ for $i=0,1, \ldots, d-1$. Since $R[0]=K[P]$ is Cohen-Macaulay, we have the exact sequence

$$
0 \longrightarrow R[i-1] \stackrel{\times \theta_{i}}{\longrightarrow} R[i-1] \longrightarrow R[i] \longrightarrow 0
$$


for $i=1,2, \ldots, d-1$. The exact sequences show that $H_{R[i]}(t)=(1-t) H_{R[i-1]}(t)$ for $i=1,2, \ldots, d-1$. Then, since $H_{K[P]}(t)=\left(h_{0}+h_{1} t+\cdots+h_{d} t^{d}\right) /(1-t)^{d}$, we have $H_{R[d-1]}(t)=\left(h_{0}+h_{1} t+\cdots+h_{d} t^{d}\right) /(1-t)$. This fact says

$$
\operatorname{dim}_{K}(R[d-1])_{k}=h_{k}+h_{k-1}+\cdots+h_{1}+h_{0}
$$

for $k=0,1, \ldots, d$. Also, since $\theta_{1}, \ldots, \theta_{d-1}, \theta_{d}^{n}$ is an h.s.o.p. of $K[P]$, the sequence

$$
0 \longrightarrow R[d-1] \stackrel{\times \theta_{d}^{n}}{\longrightarrow} R[d-1] \longrightarrow A /\left(I_{P}+\left(\theta_{1}, \ldots, \theta_{d-1}, \theta_{d}^{n}\right)\right) \longrightarrow 0
$$

is exact. Hence

$$
\begin{aligned}
\operatorname{dim}_{K}\left(A /\left(I_{P}+\left(\theta_{1}, \ldots, \theta_{d-1}, \theta_{d}^{n}\right)\right)\right)_{k} & =\operatorname{dim}_{K}(R[d-1])_{k}-\operatorname{dim}_{K}(R[d-1])_{k-n} \\
& =h_{k}+h_{k-1}+\cdots+h_{k-n+1}
\end{aligned}
$$

for $k=n, n+1, \ldots, d$, as desired.

Theorem 2.4. Let $P$ be a simplicial cell $(d-1)$-ball and $\mathbf{h}=h(P)=\left(h_{0}, h_{1}, \ldots\right.$, $\left.h_{d}\right)$. If $\partial h_{n}=0$ for some $1 \leq n \leq d-2$, then

$$
h_{k}+h_{k-1}+\cdots+h_{k-n+1} \geq \partial h_{k} \quad \text { for } k=n, n+1, \ldots, d-1 .
$$

Proof. Let $A=K\left[x_{\sigma}: \sigma \in P \backslash\{\hat{0}\}\right]$ and $J=I_{P}+\left(x_{\sigma}: \sigma \notin \partial P\right) \subset A$. Then $A / J \cong K[\partial P]$ by Lemma 2.1. For a general choice of linear forms $\theta_{1}, \ldots, \theta_{d} \in A_{1}$, we have (e.g. use a criterion of an l.s.o.p. for face rings [Ko, Lemma 6])

- $\theta_{1}, \ldots, \theta_{d-1}$ is an 1.s.o.p. of $A / J$

- $\theta_{1}, \ldots, \theta_{d-1}, \theta_{d}$ is an l.s.o.p. of $A / I_{P}$.

Let $\bar{\Theta}=\theta_{1}, \ldots, \theta_{d-1}$. Since $A / J$ is a $(d-1)$-dimensional Cohen-Macaulay ring,

$$
\operatorname{dim}_{K}(A /(J+(\bar{\Theta})))_{k}=h_{k}(\partial P)=\partial h_{k}
$$

for $k=0,1, \ldots, d-1$. In particular, since $\operatorname{dim}_{K}(A /(J+(\bar{\Theta})))_{n}=\partial h_{n}=0$ by the assumption, we have $\theta_{d}^{n} \in J+(\bar{\Theta})$. Then, since $J \supset I_{P}$, we have $J+(\bar{\Theta}) \supset$ $I_{P}+(\bar{\Theta})+\left(\theta_{d}^{n}\right)$. Thus

$$
\operatorname{dim}_{K}\left(A /\left(I_{P}+(\bar{\Theta})+\left(\theta_{d}^{n}\right)\right)\right)_{k} \geq \operatorname{dim}_{K}(A /(J+(\bar{\Theta})))_{k}=\partial h_{k}
$$

for all $k=0,1, \ldots, d-1$. Then the statement follows from Lemma 2.3 ,

2.2. Proof of (5), (6) and (7). Let $P$ be a $(d-1)$-dimensional simplicial poset. A rank 1 element of $P$ is called a vertex of $P$ and a maximal element (w.r.t. a partial order) of $P$ is called a facet of $P$. The cone of $P$ is the product of the posets

$$
C(P)=P \times\{1,2\}=\{(\sigma, i): \sigma \in P, i=1,2\},
$$

where the ordering is defined by $(\sigma, i) \geq(\tau, j)$ if $\sigma \geq \tau$ and $i \geq j$. Then $C(P)$ is simplicial and $\Gamma(C(P))$ is homeomorphic to the (topological) cone of $\Gamma(P)$. Also, a straightforward computation shows $h_{i}(P)=h_{i}(C(P))$ for all $i=0,1, \ldots, d$ and $h_{d+1}(C(P))=0$.

Let $P$ be a simplicial cell ball. We define the simplicial poset $S P$ by

$$
S P=P \cup C(\partial P) / \sim,
$$

where $\sim$ is the equivalence relation defined by $\sigma \sim(\sigma, 1)$ for all $\sigma \in \partial P$. In other words, $S P$ is a simplicial cell sphere such that $\Gamma(S P)$ is obtained from $\Gamma(P)$ by coning off its boundary.

We need the next result due to Masuda [Ma, Section 5] (see also [MR] for a simplified proof). 
Lemma 2.5 (Masuda). Let $P$ be a simplicial cell $(d-1)$-sphere, $K[P]=A / I_{P}$ the face ring of $P$ and $\Theta=\theta_{1}, \ldots, \theta_{d} \in A_{1}$ an l.s.o.p. of $K[P]$. If $x_{v_{1}} x_{v_{2}} \cdots x_{v_{d}}$ is zero in $A /\left(I_{P}+(\Theta)\right)$ for any sequence of $d$ distinct vertices $v_{1}, v_{2}, \ldots, v_{d}$ of $P$, then the number of the facets of $P$ is even.

In the rest of this section, we fix the following notation: Let $P$ be a simplicial cell $(d-1)$-ball and

$$
\mathbf{h}=h(P)=\left(h_{0}, h_{1}, \ldots, h_{d}\right) .
$$

Let $Q=C(\partial P)$ and $S P=P \cup C(\partial P) / \sim$ be simplicial posets defined as above. Also, let $A=K\left[x_{\sigma}: \sigma \in S P \backslash\{\hat{0}\}\right]$,

$$
\begin{aligned}
& J=I_{S P}+\left(x_{\sigma}: \sigma \in S P \backslash P\right)=I_{S P}+\left(x_{\sigma}: \sigma \in Q \backslash \partial P\right), \\
& L=I_{S P}+\left(x_{\sigma}: \sigma \in S P \backslash Q\right)=I_{S P}+\left(x_{\sigma}: \sigma \in P \backslash \partial P\right),
\end{aligned}
$$

and let $\Theta=\theta_{1}, \theta_{2}, \ldots, \theta_{d} \in A_{1}$ be a sequence of general linear forms. Then we have the following properties.

- $A / J \cong K[P], A / L \cong K[Q]$ and

$$
A /(L+J)=A /\left(I_{S P}+\left(x_{\sigma}: \sigma \in S P \backslash \partial P\right)\right) \cong K[\partial P] ;
$$

- $\bar{\Theta}=\theta_{1}, \ldots, \theta_{d-1}$ is an l.s.o.p. of $A /(L+J)$ and $\Theta=\theta_{1}, \ldots, \theta_{d}$ is a common l.s.o.p. of $A / I_{S P}, A / J$ and $A / L$.

Recall that $\sum_{k=0}^{d} h_{k}$ is equal to the number of the facets of $P$. The next lemma shows that, to prove (5)-(7), it is enough to study the number of the facets of $S P$.

Lemma 2.6. Suppose $\partial h_{i}=0$ for some $1 \leq i \leq d-2$. If the number of the facets of $S P$ is even, then $\sum_{k=0}^{d} h_{k}$ is even.

Proof. By the construction of $S P$, the number of the facets of $P$ is equal to that of $S P$ minus that of $\partial P$. Then the statement follows from Theorem 1.1(3).

Lemma 2.7. Let $v_{1}, v_{2}, \ldots, v_{d}$ be distinct vertices of $S P$. Then:

(i) If $\partial h_{i}=0$ for some $1 \leq i \leq d-2$, then $x_{v_{1}} x_{v_{2}} \cdots x_{v_{i}} \in L+(\Theta)$.

(ii) If $h_{j}=0$ for some $1 \leq j \leq d$, then $x_{v_{d}} x_{v_{d-1}} \cdots x_{v_{d-j+1}} \in J+(\Theta)$.

Proof. Recall $A / J \cong K[P]$ and $A / L \cong K[Q]$. Since $Q$ is the cone of $\partial P$,

$$
\operatorname{dim}_{K}(A /(L+(\Theta)))_{k}=h_{k}(Q)=h_{k}(\partial P)=\partial h_{k}
$$

for $k=0,1, \ldots, d-1$. Thus, $\partial h_{i}=0$ implies $x_{v_{1}} x_{v_{2}} \cdots x_{v_{i}} \in L+(\Theta)$. Similarly, since $\operatorname{dim}_{K}(A /(J+(\Theta)))_{j}=h_{j}, h_{j}=0$ implies $x_{v_{d}} x_{v_{d-1}} \cdots x_{v_{d-j+1}} \in J+(\Theta)$.

Now we prove (5) and (6).

Theorem 2.8. If $\partial h_{i}=0$ and $h_{j}=0$ for some positive integers $i$ and $j$ with $i+j \leq d$, then $\sum_{k=0}^{d} h_{k}$ is even.

Proof. Let $v_{1}, v_{2}, \ldots, v_{d}$ be distinct vertices of $S P$. By Lemmas 2.5] and 2.6, it is enough to prove that the monomial $x_{v_{1}} x_{v_{2}} \cdots x_{v_{d}}$ is zero in $A /\left(I_{S P}+(\Theta)\right)$.

Since $i+j \leq d$, Lemma 2.7 shows

$$
x_{v_{1}} x_{v_{2}} \cdots x_{v_{d}} \in L J+(\Theta) \subset I_{S P}+(\Theta)+\left(x_{\sigma} x_{\tau}: \sigma \in P \backslash \partial P, \tau \in Q \backslash \partial P\right) .
$$

However, for all $\sigma \in P \backslash \partial P$ and $\tau \in Q \backslash \partial P$, we have $x_{\sigma} x_{\tau} \in I_{S P}$ since $\sigma$ and $\tau$ have no common upper bounds. Thus $x_{v_{1}} x_{v_{2}} \cdots x_{v_{d}} \in I_{S P}+(\Theta)$. 
Theorem 2.9. Suppose $\partial h_{n}=0$ for some $1 \leq n<\frac{d}{2}$. If $\left(h_{\ell}+h_{\ell-1}+\cdots+\right.$ $\left.h_{\ell-n+1}\right)-\partial h_{\ell}<n$ for some $n \leq \ell \leq d-n$, then $\sum_{k=0}^{d} h_{k}$ is even.

Proof. Let $v_{1}, v_{2}, \ldots, v_{d}$ be distinct vertices of $S P$. Then $x_{v_{1}} x_{v_{2}} \cdots x_{v_{n}} \in L+(\Theta)$ by Lemma 2.7(i). Since $n+\ell \leq d$, by the same argument as in the proof of Theorem 2.8, it is enough to prove that

$$
x_{v_{d}} x_{v_{d-1}} \cdots x_{v_{d-\ell+1}} \in J+(\Theta) .
$$

Consider the exact sequence

$$
0 \longrightarrow N \longrightarrow A /\left(J+\left(\bar{\Theta}, \theta_{d}^{n}\right)\right) \longrightarrow A /\left(L+J+\left(\bar{\Theta}, \theta_{d}^{n}\right)\right) \longrightarrow 0,
$$

where $N=\left(L+J+\left(\bar{\Theta}, \theta_{d}^{n}\right)\right) /\left(J+\left(\bar{\Theta}, \theta_{d}^{n}\right)\right)$. Since $A /(L+J) \cong K[\partial P]$ and since $\bar{\Theta}$ is an l.s.o.p. of $A /(L+J)$, we have $\operatorname{dim}_{K}(A /(L+J+(\bar{\Theta})))_{n}=\partial h_{n}=0$ and

$$
A_{n}=(L+J+(\bar{\Theta}))_{n} .
$$

In particular, we have $L+J+(\bar{\Theta})=L+J+\left(\bar{\Theta}, \theta_{d}^{n}\right)$. Then, by Lemma 2.3, we have

$$
\begin{aligned}
\operatorname{dim}_{K} N_{\ell} & =\operatorname{dim}_{K}\left(A /\left(J+\left(\bar{\Theta}, \theta_{d}^{n}\right)\right)\right)_{\ell}-\operatorname{dim}_{K}\left(A /\left(L+J+\left(\bar{\Theta}, \theta_{d}^{n}\right)\right)\right)_{\ell} \\
& =\left(h_{\ell}+h_{\ell-1}+\cdots+h_{\ell-n+1}\right)-\partial h_{\ell} \\
& \leq n-1
\end{aligned}
$$

where the last inequality follows from the assumption.

Let $u_{k}=x_{v_{d}} x_{v_{d-1}} \cdots x_{v_{d-k+1}}$ for $k=1,2, \ldots, \ell$. Since $A_{n}=(L+J+(\bar{\Theta}))_{n}$, any element in $A$ of degree $n$ is contained in $L+J+(\bar{\Theta})$. Since $\ell \geq n$, this fact says that the elements

$$
u_{\ell}, u_{\ell-1} \theta_{d}, \ldots, u_{\ell-n+1} \theta_{d}^{n-1}
$$

are contained in $L+J+(\bar{\Theta})$ since they are products of elements of degree 1. Then (2.1) says that the above $n$ elements are $K$-linearly dependent in $N$. Thus there are $\alpha_{0}, \alpha_{1}, \ldots, \alpha_{n-1} \in K$ with $\left(\alpha_{0}, \alpha_{1}, \ldots, \alpha_{n-1}\right) \neq(0,0, \ldots, 0)$ such that

$$
\alpha_{0} u_{\ell}+\alpha_{1} u_{\ell-1} \theta_{d}+\cdots+\alpha_{n-1} u_{\ell-n+1} \theta_{d}^{n-1} \in J+\left(\bar{\Theta}, \theta_{d}^{n}\right) .
$$

Let $s=\min \left\{k: \alpha_{k} \neq 0\right\}$. Then there is an $h \in A$ such that

$$
\theta_{d}^{s} \alpha_{s} u_{\ell-s}+\theta_{d}^{s+1} h \in J+(\bar{\Theta}) .
$$

Since $\Theta$ is an l.s.o.p. of a Cohen-Macaulay ring $A / J, \theta_{d}$ is a non-zero divisor of $A /(J+(\bar{\Theta}))$. Thus

$$
\alpha_{s} u_{\ell-s}+\theta_{d} h \in J+(\bar{\Theta}) .
$$

This fact implies $u_{\ell-s} \in J+(\Theta)$. Since $u_{\ell-s}$ divides $u_{\ell}$, we have

$$
x_{v_{d}} x_{v_{d-1}} \cdots x_{v_{d-\ell+1}}=u_{\ell} \in J+(\Theta) \text {, }
$$

as desired.

Next, we study the condition (7). To simplify the notation, we write $R=A / J \cong$ $K[P]$ and let

$$
C=(L+J) / J=\left(\left(x_{\sigma}: \sigma \in P \backslash \partial P\right)+J\right) / J .
$$

Thus $C$ is the ideal of $R$ generated by the interior faces of $P$.

Lemma 2.10. $C$ is a d-dimensional Cohen-Macaulay $A$-module such that $H_{C}(t)=$ $\left(h_{d}+h_{d-1} t+\cdots+h_{0} t^{d}\right) /(1-t)^{d}$. 
Proof. Consider the exact sequence

$$
0 \longrightarrow C \longrightarrow R \longrightarrow R / C \longrightarrow 0 .
$$

Observe $R \cong K[P]$ and $R / C=A /(L+J) \cong K[\partial P]$. Then

$$
H_{C}(t)=H_{R}(t)-H_{R / C}(t)=H_{K[P]}(t)-H_{K[\partial P]}(t)=\frac{\left(h_{d}+h_{d-1} t+\cdots+h_{0} t^{d}\right)}{(1-t)^{d}} .
$$

Also, $C$ is a $d$-dimensional Cohen-Macaulay $A$-module since $R / C$ is a $(d-1)$ dimensional Cohen-Macaulay $A$-module and $R$ is a $d$-dimensional Cohen-Macaulay $A$-module (e.g., use [Ei, Corollary 18.6]).

Remark 2.11. The ideal $C$ is the canonical module of $R$ (see [BH] Chapter 3]). This fact can be proved in the same way as in the proof of [BH, Theorem 5.7.1], and gives an alternative proof of Lemma 2.10 .

Since $C$ is a submodule of $R$ with $\operatorname{dim} C=\operatorname{dim} R$ and since $C$ is Cohen-Macaulay, $\Theta$ is an l.s.o.p. of $C$ and we have

$$
\operatorname{dim}_{K}(C /(\Theta C))_{i}=h_{d-i}
$$

for $i=0,1, \ldots, d$ by Lemma 2.10. This fact shows

Lemma 2.12. If $h_{j}=0$ for some $1 \leq j \leq d-1$, then $C_{d-j}=\Theta C_{d-j-1}$.

Lemma 2.13. Suppose $h_{j}=0$ for some $1 \leq j \leq d-1$. If $\theta_{d} f \in(C+\bar{\Theta} R)_{d-j}$, then, for any linear form $l \in R_{1}$, one has $l f \in C+\bar{\Theta} R$.

Proof. By Lemma 2.12, $\theta_{d} f \in \bar{\Theta} R+\theta_{d} C$. Thus there is $g \in C$ such that $\theta_{d}(f-g) \in$ $\bar{\Theta} R$. Since $\theta_{d}$ is a non-zero divisor of $R /(\bar{\Theta} R)$, we have $f-g \in \bar{\Theta} R$ and $f \in C+\bar{\Theta} R$. Hence $l f \in C+\bar{\Theta} R$.

The next theorem completes the proof of the necessity of Theorem 1.2 .

Theorem 2.14. Suppose $\partial h_{i}=0$ and $h_{j}=0$ for some integers $i$ and $j$ with $0<i<\frac{d}{2}$ and $d-i<j<d$. If $\partial h_{\ell} \leq \ell$ for some $\ell \leq d-j$, then $\sum_{k=0}^{d} h_{k}$ is even.

Proof. Let $v_{1}, v_{2}, \ldots, v_{d}$ be distinct vertices of $S P$. Since $i<j$, in the same way as in the proof of Theorem 2.8, it is enough to prove that

$$
x_{v_{d}} x_{v_{d-1}} \cdots x_{v_{j+1}} \in J+(\Theta) .
$$

Let $u_{k}=x_{v_{d}} x_{v_{d-1}} \cdots x_{v_{d-k+1}}$ for $k=0,1, \ldots, d-j$, where $u_{0}=1$. We regard each $u_{k}$ as an element in $R=A / J$. Recall that $\ell$ is an integer such that $\ell \leq d-j$ and $\partial h_{\ell} \leq \ell$. Since $\bar{\Theta}$ is an l.s.o.p. of $R / C=A /(L+J) \cong K[\partial P]$,

$$
\operatorname{dim}_{K}(R /(C+\bar{\Theta} R))_{\ell}=\partial h_{\ell} \leq \ell .
$$

Then the elements

$$
u_{\ell}, u_{\ell-1} \theta_{d}, u_{\ell-2} \theta_{d}^{2}, \ldots, u_{0} \theta_{d}^{\ell} \in R_{\ell}
$$

are $K$-linearly dependent in $R /(C+\bar{\Theta} R)$. Thus there are $\alpha_{0}, \alpha_{1}, \ldots, \alpha_{\ell} \in K$ with $\left(\alpha_{0}, \alpha_{1}, \ldots, \alpha_{\ell}\right) \neq 0$ such that

$$
\alpha_{0} u_{\ell}+\alpha_{1} u_{\ell-1} \theta_{d}+\cdots+\alpha_{\ell} u_{0} \theta_{d}^{\ell} \in C+\bar{\Theta} R .
$$

Let $s=\min \left\{k: \alpha_{k} \neq 0\right\}$ and $h=\left(\sum_{k=s+1}^{\ell} \alpha_{k} u_{\ell-k} \theta_{d}^{k}\right) / \theta_{d}^{s+1}$, where $h=0$ if $s=\ell$. Then $\theta_{d}^{s}\left(\alpha_{s} u_{\ell-s}+\theta_{d} h\right) \in(C+\bar{\Theta} R)_{\ell}$ and

$$
\theta_{d}^{d-j-(\ell-s)}\left(\alpha_{s} u_{\ell-s}+\theta_{d} h\right) \in(C+\bar{\Theta} R)_{d-j} .
$$


By Lemma 2.13, we have $\left(u_{d-j} / u_{\ell-s}\right)\left(\alpha_{s} u_{\ell-s}+\theta_{d} h\right) \in C+\bar{\Theta} R$. Hence

$$
\alpha_{s} u_{d-j} \in\left(C+\bar{\Theta} R+\theta_{d} R\right)_{d-j} \subset \Theta R,
$$

where the last inclusion follows from Lemma 2.12. Since $\alpha_{s} \neq 0, u_{d-j}$ is contained in $\Theta R$. This fact shows $u_{d-j}=x_{v_{d}} x_{v_{d-1}} \cdots x_{v_{j+1}} \in J+(\Theta)$ when we regard $u_{d-j}$ as an element of $A$.

\section{Proof of SUfFicienCy}

In this section, we prove the sufficiency of Theorem 1.2

Since the proof is a bit long, we first give a short outline of the proof. The key tools of the proof are the two main lemmas outlined below.

(I) In Lemma 3.6, we introduce a simple way to make a new simplicial cell ball from a given simplicial cell ball by gluing a pair of simplexes along their boundaries.

(II) In Lemma 3.10, we construct a special simplicial cell ball whose boundary has a nice property.

Then we construct a simplicial cell ball with the desired $h$-vector as follows: First, starting from a simplex, we gradually make a larger simplicial cell ball by using (I) repeatedly. Then, at a certain point, we attach a simplicial cell ball in (II) to change the shape of the boundary. Finally, we make a simplicial cell ball with the desired $h$-vector by using (I) again. The first technique (I) appeared in [Ko]. Indeed, if $\partial \mathbf{h}$ is positive or $\sum_{k=0}^{d} h_{k}$ is even, then the first technique is enough to prove the desired statement. However, we need (II) when $\partial \mathbf{h}$ has a zero entry and $\sum_{k=0}^{d} h_{k}$ is odd.

Before proving the main lemmas, we first study what happens to the $h$-vector when we glue two simplicial posets. Two finite posets $P$ and $Q$ are said to be isomorphic if there is a bijection $f: P \rightarrow Q$, called an isomorphism, such that, for all $\sigma, \tau \in P, \sigma>\tau$ if and only if $f(\sigma)>f(\tau)$. We write $P \cong Q$ if $P$ and $Q$ are isomorphic.

Let $P$ and $Q$ be simplicial posets. Let $I \subset P$ and $J \subset Q$ be order ideals which are isomorphic as posets. For a given isomorphism $f: I \rightarrow J$, we define the equivalence relation $\sim_{f}$ on $P \cup Q$ by $\sigma \sim_{f} f(\sigma)$ for all $\sigma \in I$, and define

$$
P \cup_{f} Q=(P \cup Q) / \sim_{f} .
$$

Then $P \cup_{f} Q$ is again a simplicial poset. We write $P \cup_{I} Q$ (or $P_{I} \cup_{J} Q$ ) for this simplicial poset $P \cup_{f} Q$ when the isomorphism $f$ is clear.

Lemma 3.1. Let $P$ and $Q$ be $(d-1)$-dimensional simplicial posets and let $I \subset P$ and $J \subset Q$ be order ideals with $I \cong J$. If $\operatorname{dim} I=d-2$, then

$$
h_{k}\left(P \cup_{I} Q\right)=h_{k}(P)+h_{k}(Q)-h_{k}(I)+h_{k-1}(I)
$$

for $k=0,1, \ldots, d$, where $h_{-1}(I)=h_{d}(I)=0$.

Proof. The desired formula follows from $f_{k}\left(P \cup_{I} Q\right)=f_{k}(P)+f_{k}(Q)-f_{k}(I)$ by straightforward computations. 
For a positive integer $i$, we write $[i]=\{1,2, \ldots, i\}$. For integers $1 \leq k \leq d$, let

$$
\Delta_{d}(k)=\{F \subset[d]: F \not \supset[k]\} .
$$

By defining a partial order on $\Delta_{d}(k)$ by inclusion, we regard $\Delta_{d}(k)$ as a simplicial poset of dimension $d-2$. In other words, $\Delta_{d}(k)$ is the (abstract) simplicial complex generated by $\{[d] \backslash\{i\}: i=1,2, \ldots, k\}$. Let $\mathbf{e}_{0}, \mathbf{e}_{1}, \ldots, \mathbf{e}_{d} \in \mathbb{Z}^{d+1}$ be the unit vectors of $\mathbb{Z}^{d+1}$. Thus $\mathbf{e}_{i}$ is the vector such that the $(i+1)$-th entry of $\mathbf{e}_{i}$ is 1 and all other entries of $\mathbf{e}_{i}$ are 0 . It is easy to see that $h_{i}\left(\Delta_{d}(k)\right)=1$ for $i<k$ and $h_{i}\left(\Delta_{d}(k)\right)=0$ for $i \geq k$. Then the next lemma follows from Lemma 3.1

Lemma 3.2. With the same notation as in Lemma 3.1, if $I \cong \Delta_{d}(k)$, then

$$
h\left(P \cup_{I} Q\right)=h(P)+h(Q)-\mathbf{e}_{0}+\mathbf{e}_{k} .
$$

To apply Lemmas 3.1 and 3.2 efficiently, it is convenient to have a nice way to construct simplicial cell balls by gluing two simplicial cell balls. Constructibility and shellability give such nice ways.

Definition 3.3. A $(d-1)$-dimensional simplicial poset $P$ is said to be constructible if

(i) $P$ is a Boolean algebra of rank $d$, or

(ii) there are $(d-1)$-dimensional constructible simplicial posets $Q$ and $Q^{\prime}$ such that $P=Q_{I} \cup_{I^{\prime}} Q^{\prime}$ for some order ideals $I \subset Q$ and $I^{\prime} \subset Q^{\prime}$ and that $I \cong I^{\prime}$ is a $(d-2)$-dimensional constructible simplicial poset.

Definition 3.4. A simplicial poset is said to be pure if all its facets have the same rank. A $(d-1)$-dimensional pure simplicial poset $P$ is said to be shellable if there is an order $\sigma_{1}, \sigma_{2}, \ldots, \sigma_{r}$ of the facets of $P$, called a shelling of $P$, such that, for $i=2,3, \ldots, r,\left\langle\sigma_{1}, \sigma_{2}, \ldots, \sigma_{i-1}\right\rangle \cap\left\langle\sigma_{i}\right\rangle$ is isomorphic to $\Delta_{d}\left(k_{i}\right)$ for some $1 \leq k_{i} \leq d$.

Note that shellable simplicial posets are constructible since $\Delta_{d}(k)$ is constructible. In the rest of this paper, a simplicial cell $d$-ball which is constructible (respectively shellable) will be called a constructible d-ball (respectively shellable d-ball).

We need the following well-known properties of constructibility and shellability. See, e.g., Bj2, Theorem 11.4 and Section 12].

- Let $P$ be a simplicial cell sphere. If $Q \subsetneq P$ is a shellable simplicial poset with $\operatorname{dim} Q=\operatorname{dim} P$, then $Q$ is a $\operatorname{simplicial}$ cell ball.

- Boolean algebras and $\Delta_{d}(k)$ with $k<d$ are shellable balls.

- Let $P$ and $Q$ be constructible $d$-balls, and let $I \subsetneq \partial P$ and $J \subsetneq \partial Q$ be order ideals with $I \cong J$. If $I$ is a constructible $(d-1)$-ball, then $P_{I} \cup_{J} Q$ is a constructible $d$-ball.

For a finite set $X$, we write $\# X$ for the cardinality of $X$. The next well-known lemma, which immediately follows from Lemma 3.2 and the fact that a Boolean algebra has the $h$-vector $(1,0, \ldots, 0)$, is useful to compute $h$-vectors of shellable simplicial posets.

Lemma 3.5. Let $P$ be $a(d-1)$-dimensional shellable simplicial poset with a shelling $\sigma_{1}, \sigma_{2}, \ldots, \sigma_{r}$. Then, for $i=1,2, \ldots, d$, one has

$$
h_{i}(P)=\#\left\{\ell \in\{2,3, \ldots, r\}:\left\langle\sigma_{1}, \sigma_{2}, \ldots, \sigma_{\ell-1}\right\rangle \cap\left\langle\sigma_{\ell}\right\rangle \cong \Delta_{d}(i)\right\} .
$$

Now we prove our first main lemma. We say that a simplicial poset $P$ contains a simplicial poset $Q$ if there is an order ideal $I \subset P$ which is isomorphic to $Q$. 
Lemma 3.6. Fix integers $1 \leq i \leq m \leq d$ and $1 \leq j \leq d-i$. Let $P$ be a constructible $(d-1)$-ball such that $\partial P$ contains $\Delta_{d}(m)$ and let $\ell=\min \{m, i+j\}$. Then there is a constructible $(d-1)$-ball $Q$ such that

(i) $h(Q)=h(P)+\mathbf{e}_{i}+\mathbf{e}_{j}$, and

(ii) $\partial Q$ contains $\Delta_{d}(\ell)$.

Proof. By the assumption, there is an order ideal $L \subset \partial P$ which is isomorphic to $\Delta_{d}(m)$. We write

$$
L=\{\mathcal{L}(F): F \subset[d], F \not \supset[m]\},
$$

where the ordering is defined by $\mathcal{L}(F)>\mathcal{L}(G)$ if $F \supset G$. Since $i \leq m$,

$$
I=\{\mathcal{L}(F): F \subset[d], F \not \supset[i]\} \cong \Delta_{d}(i)
$$

is an order ideal of $\partial P$. We prepare two Boolean algebras

$$
A=\{\mathcal{A}(F): F \subset[d]\} \text { and } B=\{\mathcal{B}(F): F \subset[d]\},
$$

where the ordering is defined by inclusion on $F$. Consider the order ideal

$$
J=\{\mathcal{A}(F): F \subset[d], F \not \supset\{i+1, \ldots, i+j\}\} \cong \Delta_{d}(j) .
$$

Let

$$
Q=\left(P \cup_{I} A\right) \cup_{J} B=(P \cup A \cup B) / \sim,
$$

where $\sim$ is the equivalence relation defined by $\mathcal{L}(F) \sim \mathcal{A}(F)$ if $F \not \supset[i]$ and $\mathcal{A}(F) \sim$ $\mathcal{B}(F)$ if $F \not \supset\{i+1, \ldots, i+j\}$. Then $Q$ is a constructible $(d-1)$-ball and has the desired $h$-vector by Lemma 3.2

It remains to prove that $\partial Q$ contains $\Delta_{d}(\ell)$. Clearly, $\partial Q$ contains an order ideal

$$
\Delta=\{\mathcal{B}(F): F \subset[d], F \not \supset[i]\} \cup\{\mathcal{L}(F): F \subset[d], F \not \supset\{i+1, \ldots, \ell\}\} / \sim .
$$

We claim that $\Delta \cong \Delta_{d}(\ell)$. What we must prove is that if $F \not \supset[i]$ and $F \not \supset$ $\{i+1, \ldots, \ell\}$, then $\mathcal{L}(F) \sim \mathcal{B}(F)$. Indeed, $F \not \supset[i]$ implies $\mathcal{L}(F) \sim \mathcal{A}(F)$. Also, since $\ell \leq i+j, F \not \supset\{i+1, \ldots, \ell\}$ implies $\mathcal{A}(F) \sim \mathcal{B}(F)$.

We note that Lemma 3.6 essentially appeared in the proof of [ $\mathrm{Ko}$, Theorem 16].

Next, we develop a way to construct simplicial cell balls by applying Lemma 3.6 repeatedly. Let $\mathbf{h}=\left(h_{0}, h_{1}, \ldots, h_{d}\right) \in \mathbb{Z}_{\geq 0}^{d+1}$ be a vector such that $\sum_{k=0}^{d} h_{k}$ is even.

We write $\mathbf{h}=\mathbf{e}_{i_{1}}+\mathbf{e}_{i_{2}}+\cdots+\mathbf{e}_{i_{a}}$, where $a=\sum_{k=0}^{d} h_{k}$ and where $i_{1} \leq i_{2} \leq \cdots \leq i_{a}$. Consider the following decomposition of $\mathbf{h}$ :

$$
\mathbf{h}=\left(\mathbf{e}_{i_{1}}+\mathbf{e}_{i_{a}}\right)+\left(\mathbf{e}_{i_{2}}+\mathbf{e}_{i_{a-1}}\right)+\cdots+\left(\mathbf{e}_{i_{\frac{a}{2}}}+\mathbf{e}_{i_{\frac{a}{2}+1}}\right) .
$$

We define the initial number in (h) of $\mathbf{h}$ and the width width(h) of $\mathbf{h}$ by

$$
\operatorname{in}(\mathbf{h})=i_{\frac{a}{2}}
$$

and

$$
\operatorname{width}(\mathbf{h})=\min \left\{i_{k}+i_{a-k+1}: k=1,2, \ldots, \frac{a}{2}\right\} .
$$

The following fact is straightforward.

Lemma 3.7. With the same notation as above, one has

$$
\operatorname{width}(\mathbf{h})=\max \left\{\ell: h_{0}+h_{1}+\cdots+h_{k} \leq h_{d}+h_{d-1}+\cdots+h_{\ell-k} \text { for all } k\right\} .
$$

We need the following reformulation of Lemma 3.6 
Lemma 3.8. Let $\mathbf{h}=\left(h_{0}, h_{1}, \ldots, h_{d}\right) \in \mathbb{Z}_{\geq 0}^{d+1}$ be such that $h_{0}=0, \partial h_{i} \geq 0$ for $i=0,1, \ldots, d-1$ and $\sum_{k=0}^{d} h_{k}$ is even. Let $P$ be a constructible $(d-1)$-ball such that $\partial P$ contains $\Delta_{d}(\ell)$ with $\ell \geq \operatorname{in}(\mathbf{h})$. Then there is a constructible $(d-1)$-ball $Q$ such that $h(Q)=h(P)+\mathbf{h}$ and $\partial Q$ contains $\Delta_{d}(\min \{\ell, \operatorname{width}(\mathbf{h})\})$.

Proof. We write

$$
\mathbf{h}=\left(\mathbf{e}_{i_{1}}+\mathbf{e}_{i_{a}}\right)+\left(\mathbf{e}_{i_{2}}+\mathbf{e}_{i_{a-1}}\right)+\cdots+\left(\mathbf{e}_{i_{\frac{a}{2}}}+\mathbf{e}_{i_{\frac{a}{2}+1}}\right),
$$

where $a=\sum_{k=0}^{d} h_{k}$ and where $i_{1} \leq i_{2} \leq \cdots \leq i_{a}$. For $k=1,2, \ldots, \frac{a}{2}$, let

$$
w_{k}=\min \left\{\ell, i_{1}+i_{a}, i_{2}+i_{a-1}, \ldots, i_{k}+i_{a-k+1}\right\}
$$

and let $w_{0}=\ell$. The assumption $\partial h_{i} \geq 0$ for $i=0,1, \ldots, d-1$ implies that

$$
i_{l}+i_{a-l+1} \leq d
$$

for all $l=1,2, \ldots, \frac{a}{2}$.

We inductively prove that, for $k=0,1, \ldots, \frac{a}{2}$, there is a constructible $(d-1)$-ball $Q_{k}$ which satisfies the following two conditions:

(i) $h\left(Q_{k}\right)=h(P)+\left(\mathbf{e}_{i_{1}}+\mathbf{e}_{i_{a}}\right)+\left(\mathbf{e}_{i_{2}}+\mathbf{e}_{i_{a-1}}\right)+\cdots+\left(\mathbf{e}_{i_{k}}+\mathbf{e}_{i_{a-k+1}}\right)$;

(ii) $\partial Q_{k}$ contains $\Delta_{d}\left(w_{k}\right)$.

Since $w_{\frac{a}{2}}=\min \{\ell$, width $(\mathbf{h})\}$, if the above statement holds, then $Q_{\frac{a}{2}}$ is the simplicial poset with the desired properties.

For $k=0, Q_{0}=P$ satisfies the desired conditions. Suppose that such a $Q_{k}$, where $0 \leq k<\frac{a}{2}$, exists. We prove the existence of $Q_{k+1}$. Observe

$$
i_{k+1} \leq \min \left\{\ell, i_{a-k}\right\} \leq w_{k},
$$

where the last inequality follows from $i_{a-k} \leq i_{a-k+1} \leq \cdots \leq i_{a}$. Recall that $i_{a-k} \leq d-i_{k+1}$ by (3.1) and that $\partial Q_{k}$ contains $\Delta_{d}\left(w_{k}\right)$ by the induction hypothesis. By Lemma 3.6 (apply the case when $P=Q_{k}, i=i_{k+1}, m=w_{k}$ and $j=i_{a-k}$ ) there is a constructible $(d-1)$-ball $Q_{k+1}$ such that $h\left(Q_{k+1}\right)=h\left(Q_{k}\right)+\mathbf{e}_{i_{k+1}}+\mathbf{e}_{i_{a-k}}$ and $\partial Q_{k+1}$ contains $\Delta_{d}\left(\min \left\{w_{k}, i_{k+1}+i_{a-k}\right\}\right)=\Delta_{d}\left(w_{k+1}\right)$, as desired.

By considering the special case when $P$ is a Boolean algebra, we obtain the next corollary.

Corollary 3.9 (Kolins). Let $\mathbf{h}=\left(h_{0}, h_{1}, \ldots, h_{d}\right) \in \mathbb{Z}_{\geq 0}^{d+1}$ be such that $h_{0}=0$, $\partial h_{i} \geq 0$ for $i=0,1, \ldots, d-1$ and $\sum_{k=0}^{d} h_{k}$ is even. Then there is a constructible $(d-1)$-ball $P$ such that $h(P)=\mathbf{e}_{0}+\mathbf{h}$ and $\partial P$ contains $\Delta_{d}($ width $(\mathbf{h}))$.

The next lemma is our second main lemma.

Lemma 3.10. Let $n, m$ and $d$ be positive integers such that $n \leq \frac{d}{2}$ and $d-n \leq$ $m<d$. Let $\mathbf{h}=\left(h_{0}, h_{1}, \ldots, h_{d}\right) \in \mathbb{Z}^{d+1}$ be a vector such that $\sum_{k=0}^{d} h_{k}=d, h_{i}=1$ for $0 \leq i<d-n, h_{i}>0$ for $d-n \leq i \leq m$ and $h_{i}=0$ for $i>m$. There is a constructible $(d-1)$-ball $P$ such that

(i) $h(P)=\mathbf{h}$, and

(ii) $\partial P$ contains two ideals $I_{1}$ and $I_{2}$ that have no common facets such that $I_{1} \cong \Delta_{d}(n)$ and $I_{2} \cong \Delta_{d}(d-n)$. 
Proof. Consider the simplicial posets

$$
A=\{\mathcal{A}(F): F \subset[d+1], F \not \supset[n]\} \cong \Delta_{d+1}(n)
$$

and

$$
B=\{\mathcal{B}(F): F \subset[d+1], F \not \supset\{n+1, \ldots, d\}\} \cong \Delta_{d+1}(d-n),
$$

where the ordering is defined by inclusion on $F$. Consider the simplicial complex

$$
\begin{aligned}
\Sigma & =\langle[d+1] \backslash\{i, j\}: i \in[n], j \in\{n+1, \ldots, d\}\rangle \\
& =\{F \subset[d+1]: F \subset[d+1] \backslash\{i, j\} \text { for some } i \in[n] \text { and } j \in\{n+1, \ldots, d\}\} .
\end{aligned}
$$

Let

and

$$
I_{1}=\langle\mathcal{A}([d+1] \backslash\{i, d+1\}): i=1,2, \ldots, n\rangle \cong \Delta_{d}(n)
$$

$$
I_{2}=\langle\mathcal{B}([d+1] \backslash\{i, d+1\}): i=n+1, n+2, \ldots, d\rangle \cong \Delta_{d}(d-n) .
$$

Then

$$
\begin{aligned}
\partial A & =\langle\mathcal{A}([d+1] \backslash\{i, j\}): i=1,2, \ldots, n, j=n+1, \ldots, d+1\rangle \\
& =\{\mathcal{A}(F): F \in \Sigma\} \cup I_{1},
\end{aligned}
$$

and similarly

$$
\partial B=\{\mathcal{B}(F): F \in \Sigma\} \cup I_{2} .
$$

Let $\boldsymbol{g}=\left(g_{0}, g_{1}, \ldots, g_{d}\right)=\mathbf{h}-\sum_{i=0}^{n-1} \mathbf{e}_{i}-\sum_{i=0}^{d-n-1} \mathbf{e}_{i}$. Observe that $A$ and $B$ are constructible $(d-1)$-balls with $h(A)=\sum_{i=0}^{n-1} \mathbf{e}_{i}$ and $h(B)=\sum_{i=0}^{d-n-1} \mathbf{e}_{i}$. By Lemma 3.1. if there is a constructible $(d-2)$-ball $\Omega \subset \Sigma$ such that $-h_{i}(\Omega)+h_{i-1}(\Omega)=g_{i}$ for all $i$, then the simplicial poset

$$
P=A \cup_{\Omega} B=(A \cup B) / \sim,
$$

where $\sim$ is the equivalence relation defined by $\mathcal{A}(F) \sim \mathcal{B}(F)$ for $F \in \Omega$, satisfies the desired conditions (i) and (ii). Thus the next lemma completes the proof.

Lemma 3.11. With the same notation as in the proof of Lemma 3.10, there is a shellable $(d-2)$-ball $\Omega \subset \Sigma$ such that $-h_{i}(\Omega)+h_{i-1}(\Omega)=g_{i}$ for all $i$.

Proof. Observe

$$
\boldsymbol{g}=\left(-1, \ldots,-1,0, \ldots, 0, h_{d-n}, h_{d-n+1}, \ldots, h_{d}\right),
$$

where -1 appears in the first $n$ entries. Let

$$
\alpha_{\ell}=h_{(d-n)+\ell-1}+h_{(d-n)+\ell}+\cdots+h_{d}
$$

for $\ell=1,2, \ldots, n+1$. By the assumption on $\mathbf{h}=\left(h_{0}, h_{1}, \ldots, h_{d}\right), \alpha_{1}=n$ and $\alpha_{\ell} \leq n-(\ell-1)$ for all $\ell$.

Let

$$
\begin{aligned}
D= & \{\{p, q\}: p \in[n], q \in\{n+1, \ldots, d\}, p+q \leq d\} \\
& \cup\left[\bigcup_{\ell=1}^{m-(d-n)+1}\left\{\{p, d+\ell-p\}: p=\ell, \ell+1, \ldots, \ell+\alpha_{\ell}-1\right\}\right] .
\end{aligned}
$$

Since $\alpha_{\ell} \leq n-(\ell-1), D \subset\{\{p, q\}: p \in[n], q \in\{n+1, \ldots, d\}\}$. Let

$$
\Omega=\langle[d+1] \backslash\{p, q\}:\{p, q\} \in D\rangle \subset \Sigma .
$$

We claim that $\Omega$ satisfies the desired conditions. 
We first prove that $\Omega$ is shellable. We define the total order $\succ$ on $D$ by $\{p, q\} \succ$ $\left\{p^{\prime}, q^{\prime}\right\}$, where $p<q$ and $p^{\prime}<q^{\prime}$, if $p<p^{\prime}$ or $p=p^{\prime}$ and $q<q^{\prime}$. We show that the total order on the facets of $\Omega$ induced by $\succ$ gives a shelling of $\Omega$. Since $h_{n-d}, \ldots, h_{m}>0$ and $h_{m+1}=\cdots=h_{d}=0$ by the assumption, we have $\alpha_{1}>$ $\alpha_{2}>\cdots>\alpha_{m-(d-n)+2}=\cdots=\alpha_{n+1}=0$. Then, by the construction of $D$, for any $\{p, q\} \in D$ with $p<q$, we have $\{p-1, q\} \in D$ if $p \neq 1$ and $\{p, q-1\} \in D$ if $q \neq n+1$. This fact shows that, for any $\{p, q\} \in D$ with $\{p, q\} \neq\{1, n+1\}$,

$$
\begin{aligned}
& \langle[d+1] \backslash\{s, t\}:\{s, t\} \in D,\{s, t\} \succ\{p, q\}\rangle \cap\langle[d+1] \backslash\{p, q\}\rangle \\
& =\langle[d+1] \backslash\{p, q, k\}: k=1,2, \ldots, p-1, n+1, n+2, \ldots, q-1\rangle \\
& \cong \Delta_{d-1}(p+q-n-2) .
\end{aligned}
$$

Hence $\Omega$ is shellable. (To see that the second line contains the first line, use the fact that if $F$ is contained in the first line, then one has either $p^{\prime}=\min ([n] \backslash F)<p$ or $q^{\prime}=\min (\{n+1, \ldots, d\} \backslash F)<q$ since $[d+1] \backslash\left\{p^{\prime}, q^{\prime}\right\}$ must be contained in $\{[d+1] \backslash\{s, t\}:\{s, t\} \in D,\{s, t\} \succ\{p, q\}\}$.)

The above shelling and Lemma 3.5 show

$$
h_{i}(\Omega)=\#\{\{p, q\} \in D: p+q-n-2=i\}
$$

for $i=0,1, \ldots, d-1$. Then a simple counting shows

$$
h_{i}(\Omega)= \begin{cases}i+1, & \text { if } i \leq n-1, \\ n, & \text { if } n \leq i \leq d-n-1, \\ \alpha_{i-(d-n)+2}, & \text { if } i \geq d-n\end{cases}
$$

Note that $h_{d-n-1}(\Omega)=\alpha_{1}=n$. Then we have $-h_{i}(\Omega)+h_{i-1}(\Omega)=g_{i}$ for all $i$.

Finally, $\Omega$ is a simplicial cell $(d-2)$-ball since $\Omega$ can be identified with a $(d-2)$ dimensional subposet of a $(d-2)$-dimensional simplicial cell sphere $\partial A$.

Remark 3.12. The simplicial complex $\Sigma$ is the cone of the join of the boundaries of simplexes, and $h$-vectors of shellable subcomplexes of $\Sigma$ having the same dimension as $\Sigma$ are characterized in BFS. This will give an alternative proof of Lemma 3.11.

Theorem 3.13. If $\mathbf{h}=\left(h_{0}, h_{1}, \ldots, h_{d}\right) \in \mathbb{Z}^{d+1}$ satisfies conditions (1)-(7) in Theorem 1.2, then $\mathbf{h}$ is the h-vector of a simplicial cell $(d-1)$-ball.

Proof. If $\partial \mathbf{h}$ is positive or $\sum_{k=0}^{d} h_{k}$ is even, then the assertion was proved in $\mathrm{Ko}$, Theorem 16]. But we include a proof for completeness (our proof is essentially the same as that of Kolins).

Case 1. Suppose that all entries of $\partial \mathbf{h}$ are positive. If $\sum_{k=0}^{d} h_{k}$ is odd, then, since $\partial\left(\mathbf{h}-\mathbf{e}_{0}\right)$ is a non-negative vector, Corollary 3.9 says that $\mathbf{h}=\mathbf{e}_{0}+\left(\mathbf{h}-\mathbf{e}_{0}\right)$ is the $\mathbf{h}$-vector of a simplicial cell $(d-1)$-ball. Suppose that $\sum_{k=0}^{d} h_{k}$ is even. We write

$$
\mathbf{h}-\mathbf{e}_{0}=\left(\mathbf{e}_{i_{1}}+\mathbf{e}_{i_{a}}\right)+\left(\mathbf{e}_{i_{2}}+\mathbf{e}_{i_{a-1}}\right)+\cdots+\left(\mathbf{e}_{\frac{a-1}{2}}+\mathbf{e}_{\frac{a+3}{2}}\right)+\mathbf{e}_{i_{\frac{a+1}{2}}},
$$

where $a=\sum_{k=0}^{d} h_{k}-1$ and where $i_{1} \leq \cdots \leq i_{a}$. Let $\mathbf{h}^{\prime}=\mathbf{h}-\mathbf{e}_{0}-\mathbf{e}_{i_{\frac{a+1}{2}}}$. Since $\partial\left(\mathbf{h}-\mathbf{e}_{0}\right)$ is non-negative, we have $i_{a-l+1} \leq d-i_{l}$ for $l=1,2, \ldots, \frac{a-1}{2}$. Then $\partial \mathbf{h}^{\prime}$ is non-negative and $\operatorname{width}\left(\mathbf{h}^{\prime}\right) \geq \min \left\{i_{\frac{a+3}{2}}, \ldots, i_{a}\right\} \geq i_{\frac{a+1}{2}}$. Thus, by Corollary 3.9. there is a constructible $(d-1)$-ball $P$ such that $h(P)=\mathbf{e}_{0}+\mathbf{h}^{\prime}=\mathbf{h}-\mathbf{e}_{i_{\frac{a+1}{2}}}$ and $\partial P$ contains $\Delta_{d}\left(i_{\frac{a+1}{2}}\right)$. Let $Q$ be a simplicial cell $(d-1)$-ball obtained from $P$ by 
gluing a Boolean algebra of rank $d$ along an order ideal $J \subset \partial P$ with $J \cong \Delta_{d}\left(i_{\frac{a+1}{2}}\right)$. Lemma 3.2 guarantees that the $h$-vector of $Q$ is $h(P)+\mathbf{e}_{\frac{i^{a+1}}{2}}=\mathbf{h}$.

Case 2. Suppose that $\partial \mathbf{h}$ has a zero entry and $\sum_{k=0}^{d} h_{k}$ is even. Let

$$
n=\min \left\{k: \partial h_{k}=0\right\}
$$

and let $\mathbf{h}^{\prime}=\left(h_{0}^{\prime}, h_{1}^{\prime}, \ldots, h_{d}^{\prime}\right)=\mathbf{h}-\mathbf{e}_{0}-\mathbf{e}_{d-n}$. Note that $\mathbf{h}^{\prime}$ is a non-negative vector since $\partial h_{n-1}>0$ and $\partial h_{n}=0$ imply $h_{d-n}>0$. By the same argument as in Case 1, it is enough to prove that there is a constructible $(d-1)$-ball $P$ such that $h(P)=\mathbf{h}-\mathbf{e}_{d-n}$ and $\partial P$ contains $\Delta_{d}(d-n)$. By the choice of $n, \partial \mathbf{h}^{\prime}$ is non-negative. Thus, by Corollary 3.9 , what we must prove is $\operatorname{width}\left(\mathbf{h}^{\prime}\right) \geq d-n$.

By condition (4), for $k \geq 0$, we have

$$
h_{k+n}+\cdots+h_{k+1} \geq\left(h_{0}+h_{1}+\cdots+h_{k+n}\right)-\left(h_{d}+h_{d-1}+\cdots+h_{d-k-n}\right)
$$

(we substitute $k$ by $k+n$ in the inequality in (4)). Then we have $h_{0}+\cdots+h_{k} \leq$ $h_{d}+\cdots+h_{d-k-n}$ for all $k \geq 0$. This fact shows

$$
h_{0}^{\prime}+h_{1}^{\prime}+\cdots+h_{k}^{\prime} \leq h_{d}^{\prime}+h_{d-1}^{\prime}+\cdots+h_{d-k-n}^{\prime}
$$

for all $k \geq 0$. Then Lemma 3.7 guarantees width $\left(\mathbf{h}^{\prime}\right) \geq d-n$.

Case 3. Suppose that $\partial \mathbf{h}$ has a zero entry and $\sum_{k=0}^{d} h_{k}$ is odd. By condition (3), $d$ must be even. Let

$$
n=\min \left\{k: \partial h_{k}=0\right\} .
$$

By the symmetry of $\partial \mathbf{h}$, we have $n<\frac{d}{2}$. Let

$$
m=\min \left\{k: h_{k}=0\right\}-1 .
$$

Thus $h_{0}, h_{1}, \ldots, h_{m}>0$ and $h_{m+1}=0$. Then $m \leq d-1$ since $h_{d}=0$ and $m \geq d-n$ by condition (5).

By condition (7), $\partial h_{d-m-1} \geq d-m$. Recall that $\partial h_{n}=0, d-m \leq n$ and $\partial h_{k}>0$ for $k<n$. Then, there is a sequence of integers $d-m \leq s_{1}<s_{2}<\cdots<s_{p}=n$ such that

- $d-m>\partial h_{s_{1}}>\cdots>\partial h_{s_{p}}=0$, and

- for any $s_{i-1}<j<s_{i}$ with $i \geq 2$ one has $\partial h_{j} \geq \partial h_{s_{i-1}}$, and for any $d-m-1<j<s_{1}$ one has $\partial h_{j} \geq d-m$.

(Figure 1 explains what $s_{1}, s_{2}, \ldots, s_{p}$ are.)

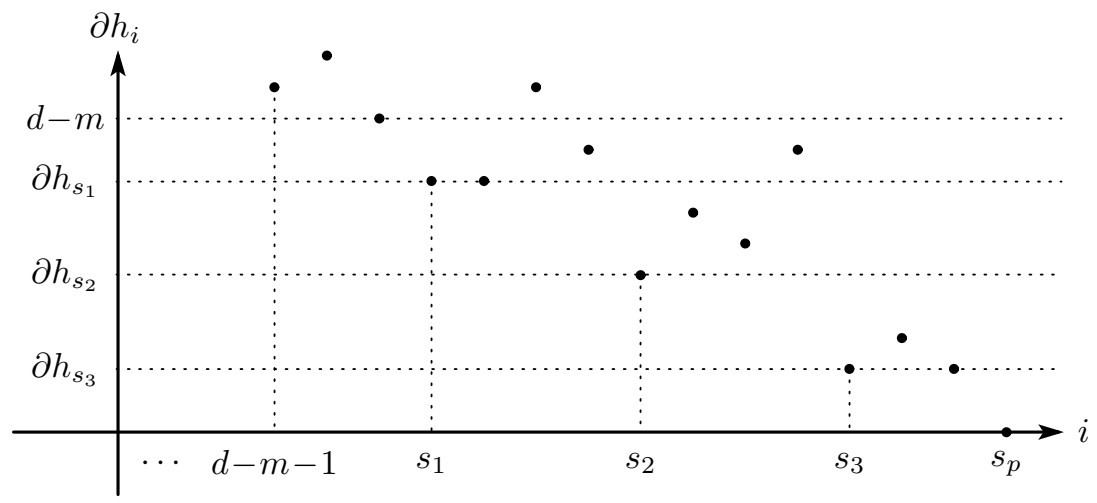

Figure 1 
Let

$$
\gamma=\left(d-m-\partial h_{s_{1}}\right) \mathbf{e}_{d-s_{1}}+\sum_{j=2}^{p}\left(\partial h_{s_{j-1}}-\partial h_{s_{j}}\right) \mathbf{e}_{d-s_{j}}
$$

and

$$
\boldsymbol{\delta}=\left(\delta_{0}, \delta_{1}, \ldots, \delta_{d}\right)=\sum_{i=0}^{m} \mathbf{e}_{i}+\gamma .
$$

By the construction of $\boldsymbol{\delta}, \sum_{k=0}^{d} \delta_{k}=d+1$ and $\delta_{d-n} \geq 2$. Let

$$
\bar{\delta}=\boldsymbol{\delta}-\mathbf{e}_{d-n} .
$$

Then $\bar{\delta}$ satisfies the assumption of Lemma 3.10. Thus there is a constructible $(d-1)$-ball $P$ such that:

- $h(P)=\overline{\boldsymbol{\delta}}$.

- $\partial P$ contains two ideals $I_{1}$ and $I_{2}$ that have no common facets such that $I_{1} \cong \Delta_{d}(n)$ and $I_{2} \cong \Delta_{d}(d-n)$.

We define $\mathbf{h}^{\prime}=\left(h_{0}^{\prime}, h_{1}^{\prime}, \ldots, h_{d}^{\prime}\right) \in \mathbb{Z}^{d+1}$ and $\mathbf{h}^{\prime \prime}=\left(h_{0}^{\prime \prime}, h_{1}^{\prime \prime}, \ldots, h_{d}^{\prime \prime}\right) \in \mathbb{Z}^{d+1}$ by

$$
\mathbf{h}^{\prime}=\left(0, \ldots, 0, h_{n+1}-1, \ldots, h_{d-n-1}-1,0, \ldots, 0\right)
$$

and

$$
\mathbf{h}^{\prime \prime}=\left(h_{0}-1, \ldots, h_{n}-1,0, \ldots, 0, h_{d-n}-1, \ldots, h_{m}-1, h_{m+1}, \ldots, h_{d}\right)-\gamma .
$$

We claim that the following conditions hold.

(i) $\mathbf{h}=\mathbf{h}^{\prime}+\mathbf{h}^{\prime \prime}+\overline{\boldsymbol{\delta}}+\mathbf{e}_{d-n}$;

(ii) $\mathbf{h}^{\prime}$ and $\mathbf{h}^{\prime \prime}$ are non-negative;

(iii) $\sum_{k=0}^{d} h_{k}^{\prime}$ and $\sum_{k=0}^{d} h_{k}^{\prime \prime}$ are even;

(iv) $\partial \mathbf{h}^{\prime}$ and $\partial \mathbf{h}^{\prime \prime}$ are non-negative and $\partial h_{n}^{\prime \prime}=0$;

(v) $\operatorname{width}\left(\mathbf{h}^{\prime}\right) \geq d-n$.

We first prove that the above conditions (i) $-(\mathrm{v})$ prove the desired statement.

By Corollary 3.9 and conditions (ii)-(v), there is a constructible $(d-1)$-ball $Q$ with $h(Q)=\mathbf{e}_{0}+\mathbf{h}^{\prime}$ such that $\partial Q$ contains an order ideal $J$ which is isomorphic to $\Delta_{d}(d-n)$. Then the simplicial poset

$$
R=Q_{J} \cup_{I_{2}} P
$$

is a constructible $(d-1)$-ball with $h(R)=\mathbf{h}^{\prime}+\overline{\boldsymbol{\delta}}+\mathbf{e}_{d-n}$ by Lemma 3.2. Also, $\partial R$ contains $\Delta_{d}(n)$ since $I_{1}$ and $I_{2}$ have no common facets.

Since $\mathbf{h}^{\prime \prime}$ is non-negative and $\sum_{k=0}^{d} h_{k}^{\prime \prime}$ is even, $\mathbf{h}^{\prime \prime}$ can be written in the form

$$
\mathbf{h}^{\prime \prime}=\left(\mathbf{e}_{i_{1}}+\mathbf{e}_{i_{a}}\right)+\left(\mathbf{e}_{i_{2}}+\mathbf{e}_{i_{a-1}}\right)+\cdots+\left(\mathbf{e}_{i_{\frac{a}{2}}}+\mathbf{e}_{i_{\frac{a}{2}+1}}\right),
$$

where $a=\sum_{k=0}^{d} h_{k}^{\prime \prime}$ and $i_{1} \leq \cdots \leq i_{a}$. Since $\partial h_{n}^{\prime \prime}=0$, we have

$$
i_{1} \leq \cdots \leq i_{\frac{a}{2}} \leq n \text { and } d-n \leq i_{\frac{a}{2}+1} \leq \cdots \leq i_{a} .
$$

Thus in $\left(\mathbf{h}^{\prime \prime}\right) \leq n$. Since $\partial R$ contains $\Delta_{d}(n)$, Lemma 3.8 guarantees the existence of a constructible $(d-1)$-ball whose $h$-vector is $h(R)+\mathbf{h}^{\prime \prime}=\mathbf{h}$.

It remains to prove (i)-(v). Statement (i) is obvious. 
Proof of (ii). Since $h_{k}>0$ for $k \leq d-n$ (see the first paragraph of Case 3), $\mathbf{h}^{\prime}$ is non-negative. To prove that $\mathbf{h}^{\prime \prime}$ is non-negative, it is enough to prove that $\delta_{k} \leq h_{k}$ for all $k$. For $k>m, h_{k} \geq 0=\delta_{k}$. Also, if $k \leq m$ and $k \notin\left\{d-s_{1}, \ldots, d-s_{p}\right\}$, then $h_{k} \geq 1=\delta_{k}$ by the choice of $m$. Suppose $k=d-s_{j}$. Since $h_{s_{j}}-h_{d-s_{j}}=$ $\partial h_{s_{j}}-\partial h_{s_{j}-1}$

$$
h_{d-s_{j}}=h_{s_{j}}+\left(\partial h_{s_{j}-1}-\partial h_{s_{j}}\right) \geq 1+\left(\partial h_{s_{j-1}}-\partial h_{s_{j}}\right)=\delta_{d-s_{j}},
$$

where the inequality follows from the choice of $s_{1}, \ldots, s_{p}$. (If $j=1$, then we consider that $\partial h_{s_{0}}=d-m$.)

Proof of (iii). Since $\partial h_{n}=\left(h_{0}+\cdots+h_{n}\right)-\left(h_{d}+\cdots+h_{d-n}\right)=0$ and since $h_{0}+h_{1}+\cdots+h_{d}$ is odd,

$$
h_{n+1}+\cdots+h_{d-n-1}=\left(h_{0}+\cdots+h_{d}\right)-\left(h_{0}+\cdots+h_{n}\right)-\left(h_{d}+\cdots+h_{d-n}\right)
$$

is odd. Then, since $d$ is even,

$$
\left(h_{0}^{\prime}+\cdots+h_{d}^{\prime}\right)=\left(h_{n+1}+\cdots+h_{d-n-1}\right)-(d-2 n-1)
$$

is even, as desired. Similarly, $\partial h_{n}=0$ implies that

$$
\left(h_{0}^{\prime \prime}+\cdots+h_{d}^{\prime \prime}\right)=\left(h_{0}+\cdots+h_{n}\right)+\left(h_{d}+\cdots+h_{d-n}\right)-2 n-2
$$

is even.

Proof of (iv). Observe $\partial h_{k}^{\prime}=\partial h_{k}-\partial h_{n}$ for $k \geq n$. Since $\partial h_{n}=0$, we have

$$
\partial h_{k}^{\prime}= \begin{cases}0, & \text { for } k=0,1, \ldots, n \\ \partial h_{k}, & \text { for } k=n+1, \ldots, \frac{d}{2}-1 .\end{cases}
$$

Hence $\partial \mathbf{h}^{\prime}$ is non-negative.

For $k=0,1, \ldots, d-m-1$,

$$
\partial h_{k}^{\prime \prime}=\partial h_{k}-(k+1) \geq 0
$$

by the condition (7). Also, for $k=n, \ldots, \frac{d}{2}-1$,

$$
\partial h_{k}^{\prime \prime}=\partial h_{n}=0 \text {. }
$$

Let $k \in\{d-m, d-m+1, \ldots, n-1\}$. Then, there is an $i$ such that $s_{i-1} \leq k<s_{i}$, where we consider that $s_{0}=d-m$ and $\partial h_{s_{0}}=d-m$. Then

$$
\begin{aligned}
\partial h_{k}^{\prime \prime} & =\partial h_{k}-(d-m)+\left\{\left(d-m-\partial h_{s_{1}}\right)+\left(\partial h_{s_{1}}-\partial h_{s_{2}}\right)+\cdots+\left(\partial h_{s_{i-2}}-\partial h_{s_{i-1}}\right)\right\} \\
& =\partial h_{k}-\partial h_{s_{i-1}} \\
& \geq 0
\end{aligned}
$$

where the last inequality follows from the choice of $s_{1}, \ldots, s_{p}$. Thus $\partial \mathbf{h}^{\prime \prime}$ is nonnegative.

Proof of (v). Condition (6) says that, for $0 \leq k \leq d-2 n$,

$$
\begin{aligned}
h_{k+n}+\cdots+h_{k+1}-n & \geq \partial h_{k+n} \\
& =\partial h_{n}+\left(h_{n+1}+\cdots+h_{n+k}\right)-\left(h_{d-n-1}+\cdots+h_{d-n-k}\right)
\end{aligned}
$$

(we substitute $\ell$ by $k+n$ in the inequality in (6)). Since $\partial h_{n}=0$, for $n<k \leq d-2 n$, we have

$$
h_{n+1}+\cdots+h_{k}+n \leq h_{d-n-1}+\cdots+h_{d-n-k} .
$$


The above inequality says

$$
\begin{aligned}
h_{0}^{\prime}+\cdots+h_{k}^{\prime} & =h_{n+1}+\cdots+h_{k}-(k-n) \\
& \leq h_{d-n-1}+\cdots+h_{d-n-k}-k=h_{d}^{\prime}+\cdots+h_{d-n-k}^{\prime}
\end{aligned}
$$

for all $n<k<d-2 n$. Also, if $k \leq n$ or $k \geq d-2 n$, then it is clear that

$$
h_{0}^{\prime}+\cdots+h_{k}^{\prime} \leq h_{d}^{\prime}+\cdots+h_{d-n-k}^{\prime} .
$$

Then Lemma 3.7 guarantees width $\left(\mathbf{h}^{\prime}\right) \geq d-n$.

\section{ACKNOWLEDGMENTS}

The author would like to thank Samuel Kolins for his valuable comments on the author's earlier work, which inspired the author to find the complete necessary and sufficient conditions, and for pointing out a mistake in an earlier draft. This work was supported by KAKENHI 22740018.

\section{REFERENCES}

[Bj1] A. Björner, Posets, regular CW complexes and Bruhat order, European J. Combin. 5 (1984), 7-16. MR746039(86e:06002)

[Bj2] A. Björner, Topological methods, In: Handbook of Combinatorics, R. Graham, M. Grotschel and L. Lovasz, Eds., Elsevier, Amsterdam, 1995, pp. 1819-1872. MR.1373690 (96m:52012)

[BFS] A. Björner, P. Frankl and R.P. Stanley, The number of faces of balanced CohenMacaulay complexes and a generalized Macaulay theorem, Combinatorica 7 (1987), 23-34. MR905148 (89d:52012)

[BH] W. Bruns and J. Herzog, Cohen-Macaulay rings, Revised Edition, Cambridge University Press, Cambridge, 1998. MR 1251956 (95h:13020)

[Ei] D. Eisenbud, Commutative algebra with a view toward algebraic geometry, Grad. Texts in Math., vol. 150, Springer-Verlag, New York, 1995. MR1322960 (97a:13001)

[Ko] S. Kolins, $f$-vectors of simplicial posets that are balls, J. Algebraic Combin., to appear.

[Ma] M. Masuda, $h$-vectors of Gorenstein* simplicial posets, Adv. Math. 194 (2005), 332-344. MR2139917 (2006b:52009)

[MMP] H. Maeda, M. Masuda and T. Panov, Torus graphs and simplicial posets, Adv. Math. 212 (2007), 458-483. MR2329309 (2008e:55007)

[MR] E. Miller and V. Reiner, Stanley's simplicial poset conjecture, after M. Masuda, Comm. Algebra 34 (2006), 1049-1053. MR2208116 (2006m:13023)

[Mu S. Murai, Face vectors of simplicial cell decompositions of manifolds, Israel J. Math., to appear.

[St1] R.P. Stanley, $f$-vectors and $h$-vectors of simplicial posets, J. Pure Appl. Algebra 71 (1991), 319-331. MR 1117642 (93b:06009)

[St2] R.P. Stanley, Combinatorics and commutative algebra, Second edition, Progr. Math., vol. 41, Birkhäuser, Boston, 1996. MR1453579(98h:05001)

Department of Mathematical Science, Faculty of Science, Yamaguchi University, 1677-1 Yoshida, YAMAGUCHI 753-8512, JAPAN

E-mail address: murai@yamaguchi-u.ac.jp 